\title{
THE VEIL
}

\section{Jocelyn Emerson}

All too soon, one must look to the surface-

and among surfaces - for a continuum residing in layers.

Recesses of color coalesce on the canvas,

all former matter of details leaking through-

until I see figuration

(residual) pressing forward between the few vertical lines-

transparent or dense, I think the idyllic pools at the peripheries of the veil-itself the very form of tending toward-(here the blue arriving at weightiness, the red at delayed fluidity.)

Assuming the white border somewhere into my sight (keep it visible, the fabric stretched, mobile) I see, meanwhile, that color has been spreading, all this time (as intended)

to its possible edges- 